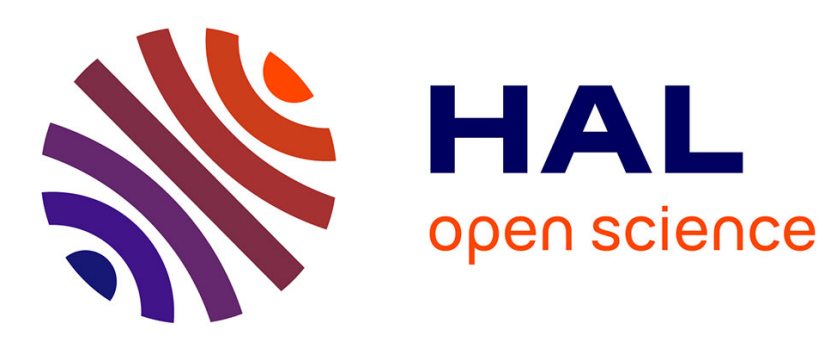

\title{
Mirrored Variants of the $(1,4)$-CMA-ES Compared on the Noisy BBOB-2010 Testbed
}

\author{
Anne Auger, Dimo Brockhoff, Nikolaus Hansen
}

\section{To cite this version:}

Anne Auger, Dimo Brockhoff, Nikolaus Hansen. Mirrored Variants of the (1,4)-CMA-ES Compared on the Noisy BBOB-2010 Testbed. GECCO workshop on Black-Box Optimization Benchmarking (BBOB'2010), Jul 2010, Portland, OR, United States. pp.1583-1590, 10.1145/1830761.1830776 . inria-00502438

\section{HAL Id: inria-00502438 \\ https://hal.inria.fr/inria-00502438}

Submitted on 14 Jul 2010

HAL is a multi-disciplinary open access archive for the deposit and dissemination of scientific research documents, whether they are published or not. The documents may come from teaching and research institutions in France or abroad, or from public or private research centers.
L'archive ouverte pluridisciplinaire HAL, est destinée au dépôt et à la diffusion de documents scientifiques de niveau recherche, publiés ou non, émanant des établissements d'enseignement et de recherche français ou étrangers, des laboratoires publics ou privés. 


\title{
Mirrored Variants of the $(1,4)$-CMA-ES Compared on the Noisy BBOB-2010 Testbed
}

\author{
[Black-Box Optimization Benchmarking Workshop]
}

\author{
Anne Auger, Dimo Brockhoff, and Nikolaus Hansen \\ Projet TAO, INRIA Saclay-lle-de-France \\ LRI, Bât 490, Univ. Paris-Sud \\ 91405 Orsay Cedex, France \\ firstname.lastname@inria.fr
}

\begin{abstract}
Derandomization by means of mirrored samples has been recently introduced to enhance the performances of $(1, \lambda)$ and $(1+2)$-Evolution-Strategies (ESs) with the aim of designing fast stochastic local search algorithms. In this paper, we investigate the impact of mirrored samples for noisy optimization. Since elitist selection is detrimental for noisy optimization, we investigate non-elitist ESs only here. We compare on the BBOB-2010 noisy benchmark testbed two variants of the $(1,4)$-CMA-ES where mirrored samples are implemented with the baseline $(1,4)$-CMA-ES. Each algorithm implements a restart mechanism. A total budget of $10^{4} \mathrm{D}$ function evaluations per trial has been used, where $D$ is the dimension of the search space.

The comparison shows that using mirroring within the $(1,4)-C M A-E S$ improves the performance in the noisy BBOB2010 scenario: the $\left(1,4_{\mathrm{m}}\right)$-CMA-ES with mirrored mutations improves significantly over the $(1,4)$-CMA-ES by $13-60 \%$ on 6 functions whereas no function with decreased performance can be reported. The $\left(1,4_{\mathrm{m}}^{\mathrm{s}}\right)$-CMA-ES, employing in addition to the mirroring a sequential selection, further improves the results over the $\left(1,4_{\mathrm{m}}\right)$-CMA-ES by additional 20 $62 \%$, depending on the function. Compared to the BBOB2009 benchmarking, the $\left(1,4_{\mathrm{m}}^{\mathrm{s}}\right)$-CMA-ES improves over the function-wise best algorithm on 7 functions with Cauchy noise type by $12-68 \%$ (in both $5 \mathrm{D}$ and $20 \mathrm{D}$ ).
\end{abstract}

\section{Categories and Subject Descriptors}

G.1.6 [Numerical Analysis]: Optimization-global optimization, unconstrained optimization; F.2.1 [Analysis of Algorithms and Problem Complexity]: Numerical Algorithms and Problems

\section{General Terms}

Algorithms

(C)ACM, 2010. This is the authors' version of the work. It is posted here by permission of ACM for your personal use. Not for redistribution. The definitive version was published at GECCO'10, July 7-11, 2010, Portland, OR, USA. http://doi.acm.org/10.1145/1830761.1830776

\section{Keywords}

Benchmarking, Black-box optimization

\section{INTRODUCTION}

Evolution Strategies (ESs) are robust stochastic search algorithms for black-box optimization where the function to be minimized, $f$, maps the continuous search space $\mathbb{R}^{D}$ into $\mathbb{R}$. ESs evolve a population of candidate solutions that are created by sampling $\lambda$ independent random vectors following a multivariate normal distribution. Recently, a new derandomization technique replacing the independent sampling of new solutions (or offspring) by mirrored samples has been introduced to enhance the performances of ESs [1]. With mirrored sampling, a single sample $\mathcal{N}$ of a multivariate normal distribution is used for two offspring of the same iteration. Denoting $X$ the current solution, the two offspring will equal $X+\mathcal{N}$ and $X-\mathcal{N}$ respectively. The resulting offspring are thus symmetric or mirrored with respect to $X$ and are thus negatively correlated. Mirrored samples have been implemented in the Covariance-Matrix-Adaptation EvolutionStrategy (CMA-ES), an ES whose characteristic is to adapt the full covariance matrix of the multivariate normal search distribution [7]. Another new concept called sequential selection was introduced together with mirrored samples [1]. Sequential selection, consists in performing sequential evaluations of the offspring and breaking the evaluation loop as soon as an offspring is better than the current solution $X$ and thus saving the remaining fitness evaluations.

In this paper, we assess quantitatively the improvement that can be brought by mirrored samples and by mirrored samples coupled with sequential selection. We compare on the BBOB-2010 noisy testbed the (1,4)-CMA-ES with two variants: first the $\left(1,4_{\mathrm{m}}\right)$-CMA-ES where mirrored samples are used, and second the $\left(1,4_{\mathrm{m}}^{\mathrm{s}}\right)$-CMA-ES that, in addition to the mirrored samples, uses sequential selection. The algorithms and the CPU timing experiments are described in a complementing paper in the same proceedings [2].

\section{RESULTS}

\subsection{Comparing $(1,4)$ - and $\left(1,4_{m}\right)$-CMA-ES}

Results from experiments comparing $(1,4)$-CMA-ES and $\left(1,4_{\mathrm{m}}\right)$-CMA-ES according to [5] on the benchmark functions given in $[4,6]$ are presented in Figures 1 and 2 and in Table 1. The expected running time (ERT), used in the figures 
and table, depends on a given target function value, $f_{\mathrm{t}}=$ $f_{\text {opt }}+\Delta f_{\mathrm{t}}$, and is computed over all relevant trials as the number of function evaluations executed during each trial while the best function value did not reach $f_{\mathrm{t}}$, summed over all trials and divided by the number of trials that actually reached $f_{\mathrm{t}}[5,8]$. Statistical significance is tested with the rank-sum test for a given target $\Delta f_{\mathrm{t}}$ using, for each trial, either the number of needed function evaluations to reach $\Delta f_{\mathrm{t}}$ (inverted and multiplied by -1 ), or, if the target was not reached, the best $\Delta f$-value achieved, measured only up to the smallest number of overall function evaluations for any unsuccessful trial under consideration.

The $(1,4 \mathrm{~m})$-CMA-ES with mirrored mutations clearly outperforms the $(1,4)$-CMA-ES. There is no function where the $\left(1,4_{\mathrm{m}}\right)$-CMA-ES shows worse results than the (1,4)-CMAES, but on all 9 functions, that can be solved by the two algorithms in $20 \mathrm{D}$, the $\left(1,4_{\mathrm{m}}\right)$-CMA-ES shows lower expected running times, of which 6 differences are statistically significant: the $\left(1,4_{\mathrm{m}}\right)$-CMA-ES is about $20 \%$ faster on $f_{101}$ and on $f_{102}, 13 \%$ faster on $f_{103}, 25 \%$ better on $f_{109}, 60 \%$ faster on $f_{118}$, and about $60 \%$ faster on $f_{121}$. On four functions, the $\left(1,4_{\mathrm{m}}\right)$-CMA-ES shows the same or even better results than the best algorithm of the BBOB-2009 benchmarking on these functions [3] $\left(f_{103}, f_{106}, f_{109}\right.$, and $\left.f_{130}\right)$ with the most remarkable improvement of $41 \%$ on the Gallagher function with Cauchy noise $\left(f_{130}\right)$ where also the $(1,4)$-CMA-ES improves over the function-wise best algorithm of BBOB2009 by $37 \%$. Note that all functions where the BBOB-2009 function-wise best algorithm is beaten comprise an underlying Cauchy noise.

\subsection{Comparing $\left(1,4_{\mathrm{m}}\right)$ - and $\left(1,4_{\mathrm{m}}^{\mathrm{s}}\right)$-CMA-ES}

The results of this comparison can be found in Fig. 3 and 4 and in Table 2 and show an even better performance if mirroring is combined with the sequential selection: the $\left(1,4_{\mathrm{m}}^{\mathrm{s}}\right)$ CMA-ES is in 20D on all 9 functions that are solved better than the $\left(1,4_{\mathrm{m}}\right)$-CMA-ES where the differences are for 8 functions statistically significant: about $20 \%$ improvement on $f_{101}$ and $f_{102}, 27 \%$ on $f_{103}, 38 \%$ on $f_{106}, 43 \%$ on $f_{109}, 62 \%$ on $f_{112}, 50 \%$ on $f_{118}$, and $56 \%$ on $f_{121}$. These improvements also result in much better expected running times than the best algorithm of the BBOB-2009 benchmarking for those functions [3], in particular on $f_{103}$ (37\% better in 20D), $f_{106}$ and $f_{118}(\geq 33 \%$ better in $5 \mathrm{D}$ and $20 \mathrm{D}), f_{121}(\geq 44 \%$ better in $5 \mathrm{D}$ and $20 \mathrm{D}), f_{109}$ and $f_{112}(30 \%$ better in $5 \mathrm{D}$ and $50 \%$ better in 20D), and $f_{130}$ (about $68 \%$ better in $5 \mathrm{D}$ and $20 \mathrm{D}$ ), see Table 2 .

\subsection{Comparing (1,4)- and $\left(1,4_{m}^{\mathrm{s}}\right)$-CMA-ES}

Due to space limitations, we refrain from showing the plots and tables of this comparison which are not too informativekeeping in mind that the $\left(1,4_{\mathrm{m}}^{\mathrm{s}}\right)$-CMA-ES already outperforms the $\left(1,4_{\mathrm{m}}\right)$-CMA-ES. Here, the $\left(1,4_{\mathrm{m}}^{\mathrm{s}}\right)$-CMA-ES, in 20D, improves over the (1,4)-CMA-ES on all 9 functions that are solved of which 8 show high statistical significance (with a $p$-value of at least 0.01 ) with a large improvement factor ranging from about $35 \%$ improvement on $f_{101-103}$, to improvement factors of $2.4\left(f_{106}\right.$ and $\left.f_{109}\right), 3.3\left(f_{118}\right)$, and more than $4\left(f_{121}\right.$ and $\left.f_{112}\right)$.

\section{CONCLUSIONS}

The idea behind derandomization by means of mirroring introduced in [1] is to use only one random sample from a multivariate normal distribution to create two (negatively correlated or mirrored) offspring. Thereby, one offspring is generated by adding a random sample to the parent solution and a second offspring then equals the solution which is symmetric to the first one with respect to the parent (by adding the negative sample to the parent). Here, this concept of mirroring has been integrated within two variants of a simple $(1,4)$-CMA-ES (of which the $\left(1,4_{\mathrm{m}}^{\mathrm{s}}\right.$ )-CMA-ES uses sequential selection [1] in addition and the $\left(1,4_{\mathrm{m}}\right)$-CMA-ES does not). The three algorithms are then compared on the noisy BBOB-2010 testbed.

Using mirroring within the $\left(1,4_{\mathrm{m}}\right)$-CMA-ES improves significantly over the $(1,4)$-CMA-ES by $13-60 \%$ on 6 functions whereas no function with decreased performance can be reported. Employing, in addition, sequential selection within the $\left(1,4_{\mathrm{m}}^{\mathrm{s}}\right)$-CMA-ES further improves the results over the $\left(1,4_{\mathrm{m}}\right)$-CMA-ES by $20-62 \%$ on 8 functions in $20 \mathrm{D}$. Important to note is that the $\left(1,4_{\mathrm{m}}^{\mathrm{s}}\right)$-CMA-ES beats the expected running time of the function-wise best algorithm of the BBOB2009 benchmarking by $30-68 \%$ on 7 functions which are all functions comprising Cauchy noise.

\section{Acknowledgments}

This work receives support by the French national research agency (ANR) within the SYSCOMM project ANR-08-SYSC017 and within the COSINUS project ANR-08-COSI-007-12.

\section{REFERENCES}

[1] A. Auger, D. Brockhoff, and N. Hansen. Mirrored sampling and sequential selection for evolution strategies. Rapport de Recherche RR-7249, INRIA Saclay-île-de-France, April 2010.

[2] A. Auger, D. Brockhoff, and N. Hansen. Mirrored variants of the $(1,4)$-CMA-ES compared on the noiseless BBOB-2010 testbed. In GECCO (Companion), 2010.

[3] A. Auger, S. Finck, N. Hansen, and R. Ros. BBOB 2009: Comparison tables of all algorithms on all noisy functions. Technical Report RT-0384, INRIA, 042010.

[4] S. Finck, N. Hansen, R. Ros, and A. Auger. Real-parameter black-box optimization benchmarking 2010: Presentation of the noisy functions. Technical Report 2009/21, Research Center PPE, 2010.

[5] N. Hansen, A. Auger, S. Finck, and R. Ros. Real-parameter black-box optimization benchmarking 2010: Experimental setup. Technical Report RR-7215, INRIA, 2010.

[6] N. Hansen, S. Finck, R. Ros, and A. Auger. Real-parameter black-box optimization benchmarking 2009: Noisy functions definitions. Technical Report RR-6869, INRIA, 2009. Updated February 2010.

[7] N. Hansen, S. D. Müller, and P. Koumoutsakos. Reducing the time complexity of the derandomized evolution strategy with covariance matrix adaptation. Evolutionary Computation, 11(1):1-18, 2003.

[8] K. Price. Differential evolution vs. the functions of the second ICEO. In Proceedings of the IEEE International Congress on Evolutionary Computation, pages 153-157, 1997. 
101 Sphere (moderate) 104 Rosenbrock (moderate)

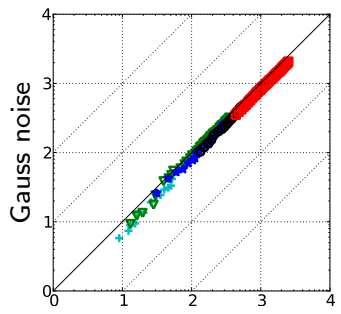

102 Sphere (moderate)

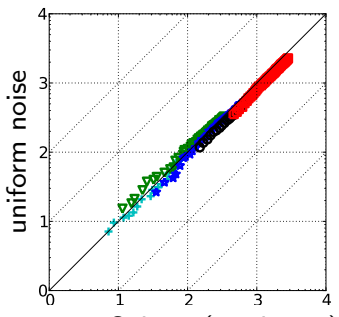

103 Sphere (moderate)

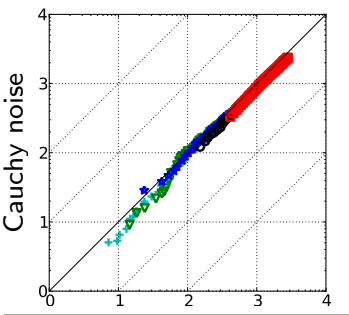

116 Ellipsoid

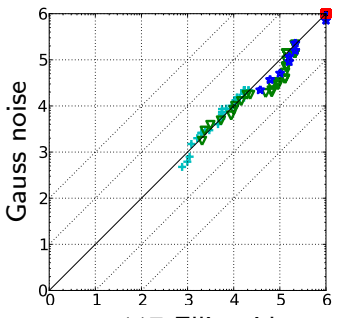

117 Ellipsoid

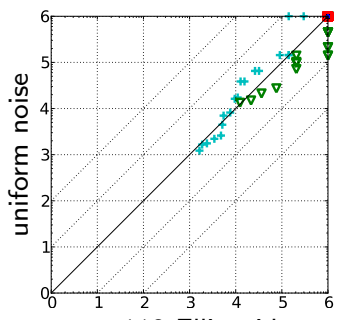

118 Ellipsoid

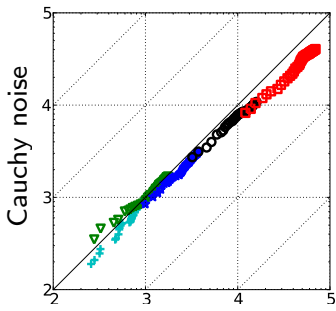

107 Sphere

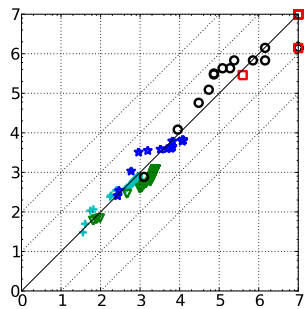

105 Rosenbrock (moderate)

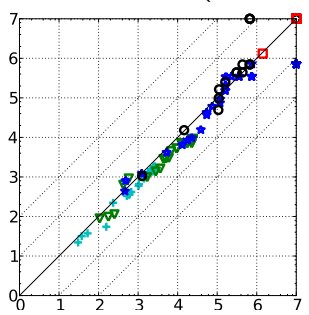

106 Rosenbrock (moderate)
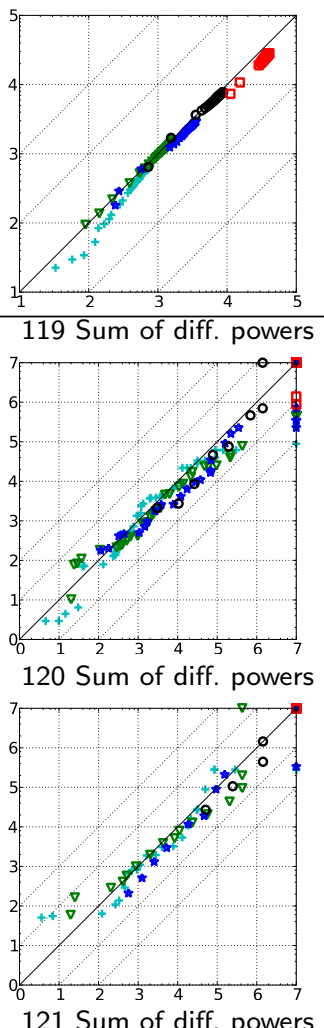

121 Sum of diff. powers

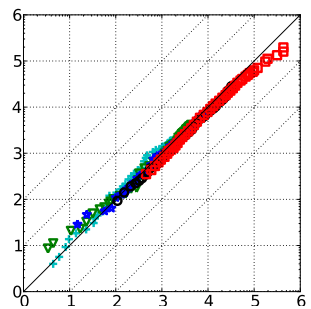

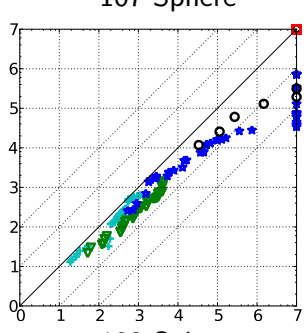

108 Sphere

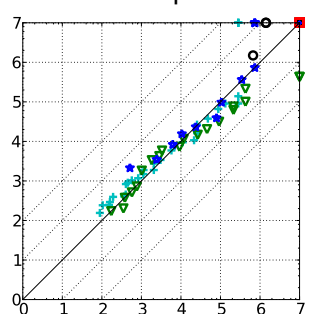

109 Sphere

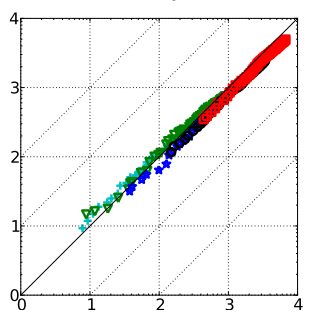

122 Schaffer F7

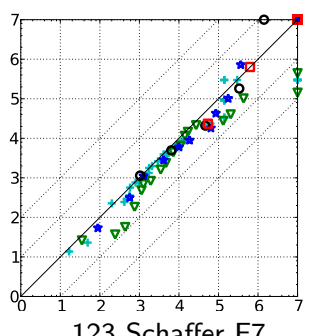

123 Schaffer F7

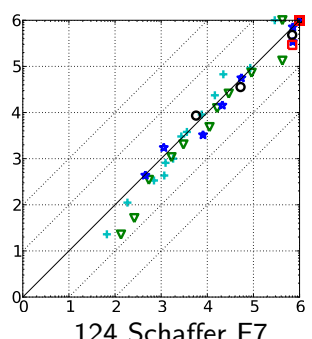

124 Schaffer F7

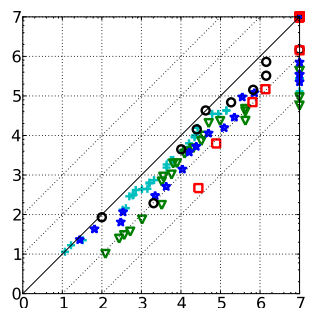

110 Rosenbrock

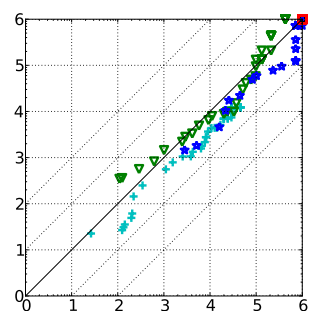

111 Rosenbrock

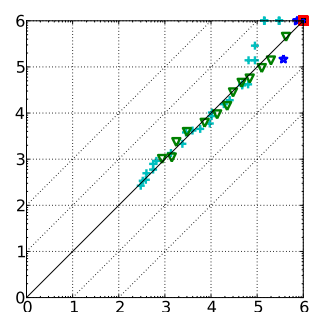

112 Rosenbrock

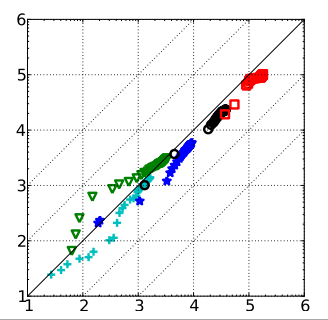

125 Griewank-Rosenbrock

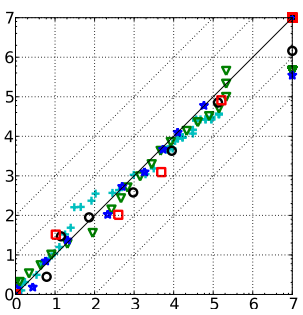

126 Griewank-Rosenbrock

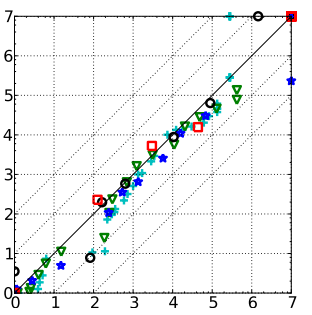

127 Griewank-Rosenbrock

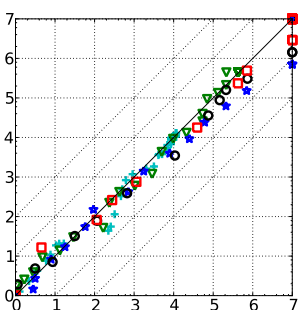

113 Step-ellipsoid

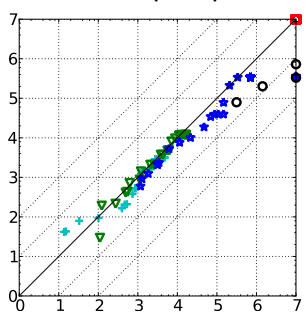

114 Step-ellipsoid

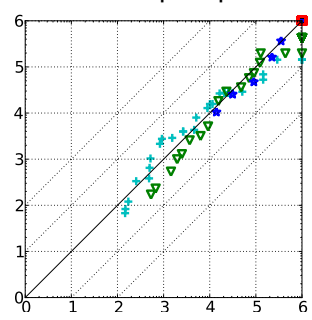

115 Step-ellipsoid

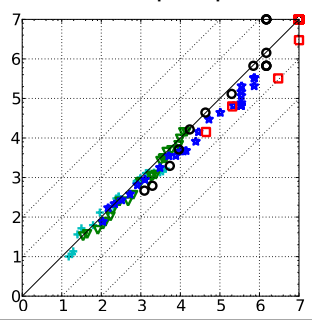

128 Gallagher

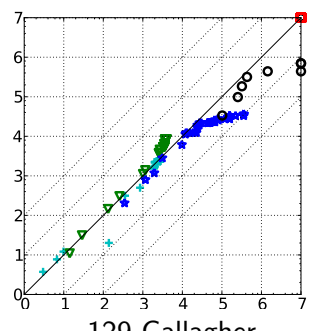

129 Gallagher

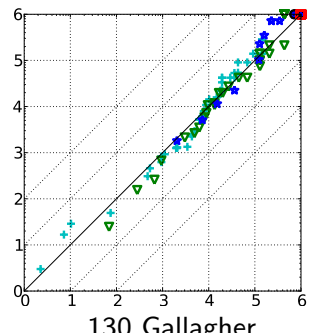

130 Gallagher

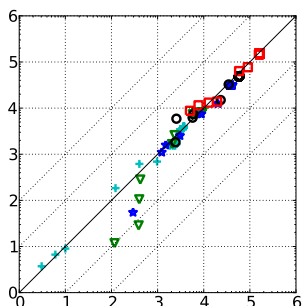

Figure 1: Expected running time (ERT in log10 of number of function evaluations) of (1,4 $4_{\mathrm{m}}$-CMA-ES versus $(1,4)$-CMA-ES for 46 target values $\Delta f \in\left[10^{-8}, 10\right]$ in each dimension for functions $f_{101}-f_{130}$. Markers on the upper or right edge indicate that the target value was never reached by $(1,4 \mathrm{~m})$-CMA-ES or (1,4)-CMA-ES respectively. Markers represent dimension: 2: $+, 3: \nabla, 5: \star, 10: \circ, 20: \square$. 
$5-\mathrm{D}$

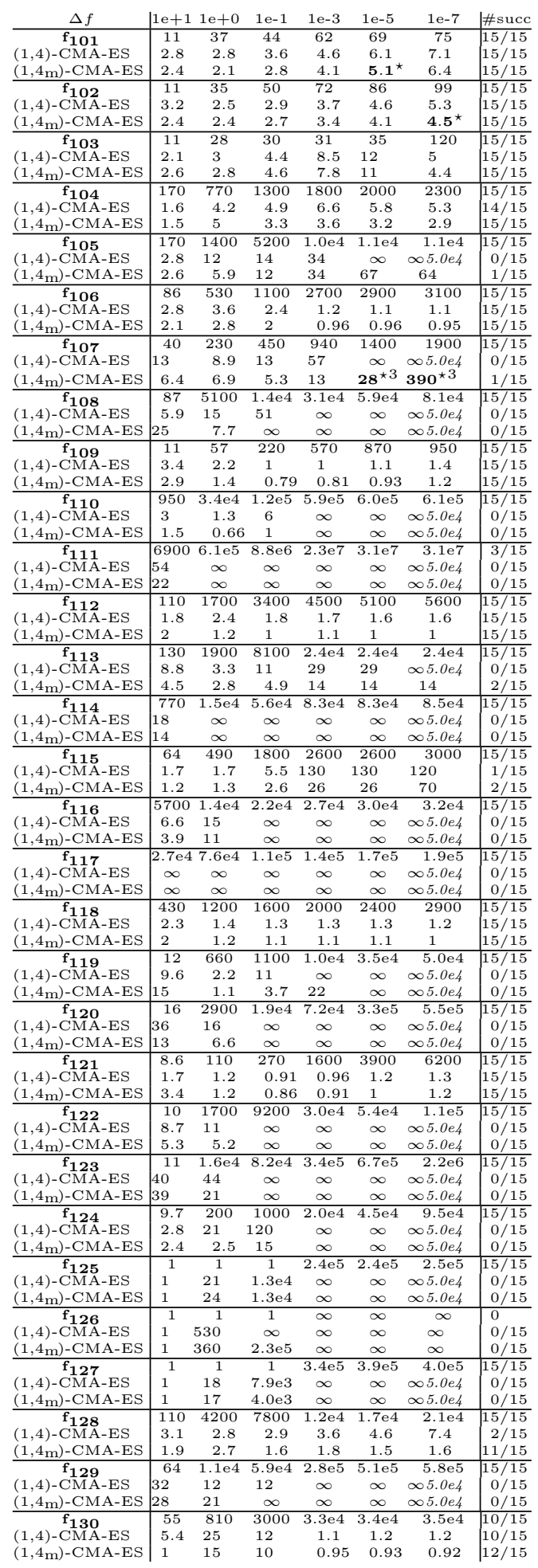

20-D

\begin{tabular}{|c|c|c|c|c|c|c|c|}
\hline$\Delta f$ & $1 \mathrm{e}+1$ & $1 \mathrm{e}+0$ & $1 \mathrm{e}-1$ & $1 \mathrm{e}-3$ & $1 e-5$ & $1 e-7$ & \#succ \\
\hline$f_{101}$ & 59 & 360 & 510 & 700 & 740 & 780 & $15 / 15$ \\
\hline$(1,4)$-CMA-ES & 7.4 & 1.8 & 1.7 & 1.9 & 2.5 & 2.9 & $15 / 15$ \\
\hline$(1,4 \mathrm{~m})$-CMA-ES & 5.9 & 1.5 & $1.4^{\star}$ & $1.6^{\star 2}$ & $\mathbf{2}^{\star 3}$ & $2.4^{\star 3}$ & $15 / 15$ \\
\hline$f_{102}$ & 230 & 400 & 580 & 920 & 1200 & 1400 & $15 / 15$ \\
\hline$(1,4)-\mathrm{CMA}-\mathrm{ES}$ & 2 & 1.8 & 1.6 & 1.6 & 1.7 & 1.8 & $15 / 15$ \\
\hline$(1,4 \mathrm{~m})$-CMA-ES & 1.5 & $1.4^{\star 2}$ & $21.3^{\star 2}$ & $1.3^{\star 2}$ & $1.4^{\star 2}$ & $1.4^{\star 2}$ & $15 / 15$ \\
\hline$f_{103}$ & 65 & 420 & 630 & 1300 & 1900 & 2500 & $14 / 15$ \\
\hline$(1,4)-C M A-E S$ & 6.3 & 1.5 & 1.4 & 1.1 & 1 & 1 & $15 / 15$ \\
\hline$(1,4 \mathrm{~m})$-CMA-ES & $5.1^{\star}$ & $1.3^{\star}$ & $1.2^{\star 2}$ & $0.91^{\star 2}$ & $0.88^{\star 3 \downarrow}$ & $30.87^{\star} \star 3 \downarrow$ & $15 / 15$ \\
\hline$f_{104}$ & $2.4 \mathrm{e} 4$ & $8.6 \mathrm{e} 4$ & $1.7 \mathrm{e} 5$ & $1.8 \mathrm{e} 5$ & $1.9 \mathrm{e} 5$ & $2.0 \mathrm{e} 5$ & $15 / 15$ \\
\hline 1,4$)-C M A-E S$ & 17 & $\infty$ & $\infty$ & $\infty$ & $\infty$ & $\infty 2.0 \mathrm{e} 5$ & $0 / 15$ \\
\hline$(1,4 \mathrm{~m})$-CMA-ES & 12 & $\infty$ & $\infty$ & $\infty$ & $\infty$ & $\infty 2.0 \mathrm{e} 5$ & $0 / 15$ \\
\hline$f_{105}$ & $1.9 \mathrm{e} 5$ & $6.1 \mathrm{e} 5$ & $6.3 \mathrm{e} 5$ & $6.5 \mathrm{e} 5$ & $6.6 \mathrm{e} 5$ & $6.7 \mathrm{e} 5$ & $15 / 15$ \\
\hline 1,4)-CMA-ES & 7.4 & $\infty$ & $\infty$ & $\infty$ & $\infty$ & $\infty 2.0 e 5$ & $0 / 15$ \\
\hline$(1,4 \mathrm{~m})$-CMA-ES & 6.9 & $\infty$ & $\infty$ & $\infty$ & $\infty$ & $\infty 2.0 e 5$ & $0 / 15$ \\
\hline$f_{106}$ & $1.1 \mathrm{e} 4$ & $2.2 \mathrm{e} 4$ & $2.4 \mathrm{e} 4$ & $2.5 \mathrm{e} 4$ & $2.6 \mathrm{e} 4$ & $2.7 \mathrm{e} 4$ & $15 / 15$ \\
\hline$(1,4)-C M A-E S$ & 0.97 & 1.5 & 1.5 & 1.5 & 1.5 & 1.5 & $15 / 15$ \\
\hline$\left(1,4_{\mathrm{m}}\right)$-CMA-ES & $0.64^{\downarrow 2}$ & 1 & 1 & 1 & 1 & 1 & $15 / 15$ \\
\hline$f_{107}$ & 8600 & $1.4 \mathrm{e} 4$ & $1.6 \mathrm{e} 4$ & $2.7 \mathrm{e} 4$ & $5.2 \mathrm{e} 4$ & $6.5 \mathrm{e} 4$ & $15 / 15$ \\
\hline )-CMA-ES & $\infty$ & $\infty$ & $\infty$ & $\infty$ & $\infty$ & $\infty 2.0 \mathrm{e} 5$ & $0 / 15$ \\
\hline $1,4 \mathrm{~m})$-CMA-ES & $\infty$ & $\infty$ & $\infty$ & $\infty$ & $\infty$ & $\infty 2.0 e 5$ & $0 / 15$ \\
\hline$f_{108}$ & $5.8 \mathrm{e} 4$ & $9.7 \mathrm{e} 4$ & $2.0 \mathrm{e} 5$ & $4.5 \mathrm{e} 5$ & $6.3 \mathrm{e} 5$ & $9.0 \mathrm{e} 5$ & $15 / 15$ \\
\hline -CMA-ES & $\infty$ & $\infty$ & $\infty$ & $\infty$ & $\infty$ & $\infty 2.0 e 5$ & $0 / 15$ \\
\hline, $4 \mathrm{~m})-\mathrm{CMA}-\mathrm{ES}$ & $\infty$ & $\infty$ & $\infty$ & $\infty$ & $\infty$ & $\infty 2.0 e 5$ & $0 / 15$ \\
\hline$f_{109}$ & 330 & 630 & 1100 & 2300 & 3600 & 5000 & $15 / 15$ \\
\hline 1,4$)-C M A-E S$ & 1.3 & 1.4 & 1.3 & 1.1 & 1.2 & 1.2 & $15 / 15$ \\
\hline$(1,4 \mathrm{~m})$-CMA-ES & $1.1^{\star}$ & $1.1^{\star}$ & $1^{\star}$ & $0.95 \star 2$ & $0.91 \star 3$ & $0.88^{\star 2 \downarrow}$ & $15 / 15$ \\
\hline$f_{110}$ & $\infty$ & $\infty$ & $\infty$ & $\infty$ & $\infty$ & $\infty$ & \\
\hline )-CMA-ES & $\infty$ & $\infty$ & $\infty$ & $\infty$ & $\infty$ & $\infty$ & $0 / 15$ \\
\hline 1m)-CMA-ES & $\infty$ & $\infty$ & $\infty$ & $\infty$ & $\infty$ & $\infty$ & $0 / 15$ \\
\hline$f_{111}$ & $\infty$ & $\infty$ & $\infty$ & $\infty$ & $\infty$ & $\infty$ & \\
\hline -CMA-ES & $\infty$ & $\infty$ & $\infty$ & $\infty$ & $\infty$ & $\infty$ & $0 / 15$ \\
\hline 4m)-CMA-ES & $\infty$ & $\infty$ & $\infty$ & $\infty$ & $\infty$ & $\infty$ & $0 / 15$ \\
\hline$f_{112}$ & $2.6 \mathrm{e} 4$ & $6.4 \mathrm{e} 4$ & $7.0 \mathrm{e} 4$ & $7.4 \mathrm{e} 4$ & $7.6 \mathrm{e} 4$ & $7.8 \mathrm{e} 4$ & $15 / 15$ \\
\hline 4)-CMA-ES & 1.4 & 1.6 & 2.1 & 2.3 & 2.3 & 2.3 & $11 / 15$ \\
\hline $1,4 \mathrm{~m})$-CMA-ES & $0.75^{\star}$ & 1.2 & 1.3 & 1.3 & 1.3 & 1.3 & $14 / 15$ \\
\hline$f_{113}$ & $5.0 \mathrm{e} 4$ & $3.6 \mathrm{e} 5$ & $5.6 \mathrm{e} 5$ & $5.9 \mathrm{e} 5$ & $5.9 \mathrm{e} 5$ & $5.9 \mathrm{e} 5$ & $15 / 15$ \\
\hline -CMA-ES & $\infty$ & $\infty$ & $\infty$ & $\infty$ & $\infty$ & $\infty 2.0 e 5$ & $0 / 15$ \\
\hline $1,4 \mathrm{~m})$-CMA-ES & $\infty$ & $\infty$ & $\infty$ & $\infty$ & $\infty$ & $\infty 2.0 \mathrm{e} 5$ & $0 / 15$ \\
\hline$f_{114}$ & $2.1 \mathrm{e} 5$ & $1.1 \mathrm{e} 6$ & $1.4 \mathrm{e} 6$ & $1.6 \mathrm{e} 6$ & $1.6 \mathrm{e} 6$ & $1.6 \mathrm{e} 6$ & $15 / 15$ \\
\hline -CMA-ES & $\infty$ & $\infty$ & $\infty$ & $\infty$ & $\infty$ & $\infty 2.0 e 5$ & $0 / 15$ \\
\hline$(1,4 \mathrm{~m})-\mathrm{CMA}-\mathrm{ES}$ & $\infty$ & $\infty$ & $\infty$ & $\infty$ & $\infty$ & $\infty 2.0 e 5$ & $0 / 15$ \\
\hline$f_{115}$ & 2400 & $3.0 \mathrm{e} 4$ & $9.2 \mathrm{e} 4$ & $1.3 \mathrm{e} 5$ & $1.3 \mathrm{e} 5$ & $1.3 \mathrm{e} 5$ & $15 / 15$ \\
\hline )-CMA-ES & 18 & $\infty$ & $\infty$ & $\infty$ & $\infty$ & $\infty 2.0 e 5$ & $0 / 15$ \\
\hline$\left(1,4_{\mathrm{m}}\right)$-CMA-ES & 5.9 & $\infty$ & $\infty$ & $\infty$ & $\infty$ & $\infty 2.0 e 5$ & $0 / 15$ \\
\hline$f_{116}$ & $5.0 \mathrm{e} 5$ & $6.9 \mathrm{e} 5$ & $8.9 \mathrm{e} 5$ & $1.0 \mathrm{e} 6$ & $1.1 \mathrm{e} 6$ & $1.1 \mathrm{e} 6$ & $15 / 15$ \\
\hline )-CMA-ES & $\infty$ & $\infty$ & $\infty$ & $\infty$ & $\infty$ & $\infty 2.0 e 5$ & $0 / 15$ \\
\hline$(1,4 \mathrm{~m})$-CMA-ES & $\infty$ & $\infty$ & $\infty$ & $\infty$ & $\infty$ & $\infty 2.0 \mathrm{e} 5$ & $0 / 15$ \\
\hline$f_{117}$ & $1.8 \mathrm{e} 6$ & $2.5 \mathrm{e} 6$ & $2.6 \mathrm{e} 6$ & $2.9 \mathrm{e} 6$ & $3.2 \mathrm{e} 6$ & $3.6 \mathrm{e} 6$ & $15 / 15$ \\
\hline -CMA-ES & $\infty$ & $\infty$ & $\infty$ & $\infty$ & $\infty$ & $\infty 2.0 e 5$ & $0 / 15$ \\
\hline$\left(1,4_{\mathrm{m}}\right)-\mathrm{CMA}-\mathrm{ES}$ & $\infty$ & $\infty$ & $\infty$ & $\infty$ & $\infty$ & $\infty 2.0 \mathrm{e} 5$ & $0 / 15$ \\
\hline$f_{118}$ & 6900 & $1.2 \mathrm{e} 4$ & $1.8 \mathrm{e} 4$ & $2.6 \mathrm{e} 4$ & $3.0 \mathrm{e} 4$ & $3.3 \mathrm{e} 4$ & $15 / 15$ \\
\hline$(1,4)$-CMA-ES & 1.8 & 1.9 & 2.1 & 1.9 & 2 & 22 & $15 / 15$ \\
\hline$(1,4 \mathrm{~m})-\mathrm{CMA}-\mathrm{ES}$ & 1.2 & 1.2 & $1.2^{\star 2}$ & $1.2 \star 3$ & $1.2^{\star 3}$ & $1.2^{\star 3}$ & $15 / 15$ \\
\hline$f_{119}$ & 2800 & $2.9 \mathrm{e} 4$ & $3.6 \mathrm{e} 4$ & $4.1 \mathrm{e} 5$ & $1.4 \mathrm{e} 6$ & $1.9 \mathrm{e} 6$ & $15 / 15$ \\
\hline ,4)-CMA-ES & $\infty$ & $\infty$ & $\infty$ & $\infty$ & $\infty$ & $\infty 2.0 e 5$ & $0 / 15$ \\
\hline$(1,4 \mathrm{~m})$-CMA-ES & 320 & $\infty$ & $\infty$ & $\infty$ & $\infty$ & $\infty 2.0 e 5$ & $0 / 15$ \\
\hline$f_{120}$ & $3.6 \mathrm{e} 4$ & $1.8 \mathrm{e} 5$ & $2.8 \mathrm{e} 5$ & $1.6 \mathrm{e} 6$ & $6.7 \mathrm{e} 6$ & $1.4 \mathrm{e} 7$ & $13 / 15$ \\
\hline ,4)-CMA-ES & $\infty$ & $\infty$ & $\infty$ & $\infty$ & $\infty$ & $\infty 2.0 e 5$ & $0 / 15$ \\
\hline $4 \mathrm{~m})$-CMA-ES & $\infty$ & $\infty$ & $\infty$ & $\infty$ & $\infty$ & $\infty 2.0 e 5$ & $0 / 15$ \\
\hline$f_{121}$ & 250 & 770 & 1400 & 9300 & $3.4 \mathrm{e} 4$ & $5.7 \mathrm{e} 4$ & $15 / 15$ \\
\hline$(1,4)$-CMA-ES & 1.8 & 1.5 & 1.4 & $0.87^{\downarrow 2}$ & & 2.1 & $6 / 15$ \\
\hline ,4m)-CMA-ES & 1.4 & 1.1 & $1 \star 2$ & $0.73 \downarrow 2$ & $0.75 \star \downarrow 2$ & $1.2^{\star}$ & $10 / 15$ \\
\hline$f_{122}$ & 690 & $5.2 \mathrm{e} 4$ & $1.4 \mathrm{e} 5$ & $7.9 \mathrm{e} 5$ & $2.0 \mathrm{e} 6$ & $5.8 \mathrm{e} 6$ & $15 / 15$ \\
\hline ,4)-CMA-ES & 78 & $\infty$ & $\infty$ & $\infty$ & $\infty$ & $\infty 2.0 e 5$ & $0 / 15$ \\
\hline$(1,4 \mathrm{~m})$-CMA-ES & 34 & $\infty$ & $\infty$ & $\infty$ & $\infty$ & $\infty 2.0 e 5$ & $0 / 15$ \\
\hline & 1100 & $5.3 \mathrm{e} 5$ & $1.5 \mathrm{e} 6$ & $5.3 \mathrm{e} 6$ & $2.7 \mathrm{e} 7$ & $1.6 \mathrm{e} 8$ & 0 \\
\hline ,4)-CMA-ES & 650 & $\infty$ & $\infty$ & $\infty$ & $\infty$ & $\infty 2.0 e 5$ & $0 / 15$ \\
\hline $4 \mathrm{~m})$-CMA-ES & 280 & $\infty$ & $\infty$ & $\infty$ & $\infty$ & $\infty 2.0 e 5$ & $0 / 15$ \\
\hline$f_{124}$ & 190 & 2000 & $4.1 \mathrm{e} 4$ & $1.3 \mathrm{e} 5$ & $3.9 \mathrm{e} 5$ & $8.0 \mathrm{e} 5$ & $15 / 15$ \\
\hline 4)-CMA-ES & 140 & $\infty$ & $\infty$ & $\infty$ & $\infty$ & $\infty 2.0 e 5$ & $0 / 15$ \\
\hline (m)-CMA-ES & 2.4 & $\infty$ & $\infty$ & $\infty$ & $\infty$ & $\infty 2.0 \mathrm{e} 5$ & $0 / 15$ \\
\hline$f_{125}$ & 1 & 1 & 1 & $2.5 \mathrm{e} 7$ & $8.0 \mathrm{e} 7$ & $8.1 \mathrm{e} 7$ & $4 / 15$ \\
\hline 4)-CMA-ES & 1 & $1.6 \mathrm{e} 5$ & $\infty$ & $\infty$ & $\infty$ & $\infty 2.0 e 5$ & $0 / 15$ \\
\hline m)-CMA-ES & 1 & $8.1 \mathrm{e} 4$ & $\infty$ & $\infty$ & $\infty$ & $\infty 2.0 e 5$ & $0 / 15$ \\
\hline$f_{126}$ & 1 & 1 & 1 & $\infty$ & $\infty$ & $\infty$ & 0 \\
\hline -CMA-ES & 1 & $\infty$ & $\infty$ & $\infty$ & $\infty$ & $\infty$ & $0 / 15$ \\
\hline m)-CMA-ES & 1 & $\infty$ & $\infty$ & $\infty$ & $\infty$ & $\infty$ & $0 / 15$ \\
\hline & 1 & 1 & 1 & $4.4 \mathrm{e} 6$ & $7.3 \mathrm{e} 6$ & $7.4 \mathrm{e} 6$ & $15 / 15$ \\
\hline )-CMA-ES & 1 & $1.1 \mathrm{e} 3$ & $\infty$ & $\infty$ & $\infty$ & $\infty 2.0 e 5$ & $0 / 15$ \\
\hline 4m)-CMA-ES & 1 & 760 & $2.9 \mathrm{e} 6$ & $\infty$ & $\infty$ & $\infty 2.0 e 5$ & $0 / 15$ \\
\hline$f_{128}$ & & $1.3 \mathrm{e} 7$ & $1.7 \mathrm{e} 7$ & $1.7 \mathrm{e} 7$ & $1.7 \mathrm{e} 7$ & $1.7 \mathrm{e} 7$ & $9 / 15$ \\
\hline 4)-CMA-ES & $\infty$ & $\infty$ & $\infty$ & $\infty$ & $\infty$ & $\infty 2.0 e 5$ & $0 / 15$ \\
\hline $4 \mathrm{~m})$-CMA-ES & $\infty$ & $\infty$ & $\infty$ & $\infty$ & $\infty$ & $\infty 2.0 \mathrm{e} 5$ & $0 / 15$ \\
\hline$f_{129}$ & $7.8 \mathrm{e} 6$ & $4.1 \mathrm{e} 7$ & $4.2 \mathrm{e} 7$ & $4.2 \mathrm{e} 7$ & $4.2 \mathrm{e} 7$ & $4.2 \mathrm{e} 7$ & $5 / 15$ \\
\hline ,4)-CMA-ES & $\infty$ & $\infty$ & $\infty$ & $\infty$ & $\infty$ & $\infty 2.0 e 5$ & $0 / 15$ \\
\hline$(1,4 \mathrm{~m})$-CMA-ES & $\infty$ & $\infty$ & $\infty$ & $\infty$ & $\infty$ & $\infty 2.0 e 5$ & $0 / 15$ \\
\hline$f_{130}$ & 4900 & $9.3 \mathrm{e} 4$ & $2.5 \mathrm{e} 5$ & $2.5 \mathrm{e} 5$ & $2.6 \mathrm{e} 5$ & $2.6 \mathrm{e} 5$ & $7 / 15$ \\
\hline $\begin{array}{l}1,4) \text {-CMA-ES } \\
1,4 m \text { )-CMA-ES }\end{array}$ & $\begin{array}{l}1 \\
1.8\end{array}$ & $\begin{array}{l}0.97 \\
0.83\end{array}$ & $\begin{array}{l}0.63 \\
0.59\end{array}$ & $\begin{array}{l}0.63 \\
0.59\end{array}$ & $\begin{array}{l}0.63 \\
0.59\end{array}$ & $\begin{array}{l}0.63 \\
0.59\end{array}$ & $\mid \begin{array}{l}11 / 15 \\
11 / 15\end{array}$ \\
\hline
\end{tabular}

Table 1: ERT in number of function evaluations divided by the best ERT measured during BBOB-2009 (given in the respective first row) for the algorithms $(1,4)$-CMA-ES and $\left(1,4_{\mathrm{m}}\right)$-CMA-ES for different $\Delta f$ values for functions $f_{101}-f_{130}$. The median number of conducted function evaluations is additionally given in italics, if $\operatorname{ERT}\left(10^{-7}\right)=\infty$. \#succ is the number of trials that reached the final target $f_{\mathrm{opt}}+10^{-8}$. Bold entries are statistically significantly better compared to the other algorithm, with $p=0.05$ or $p=10^{-k}$ where $k>1$ is the number following the $\star$ symbol, with Bonferroni correction of 60 . 
$5-\mathrm{D}$
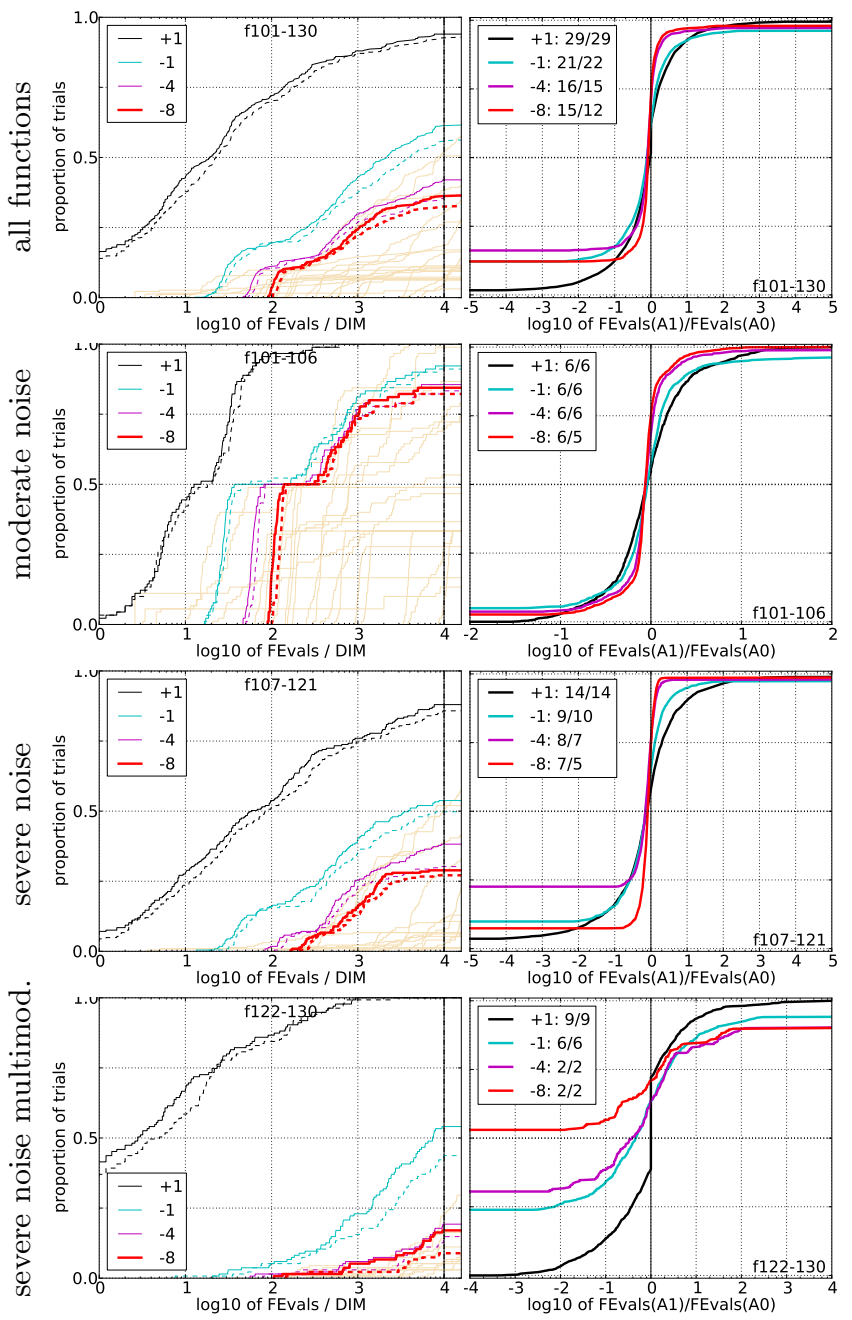

$20-\mathrm{D}$
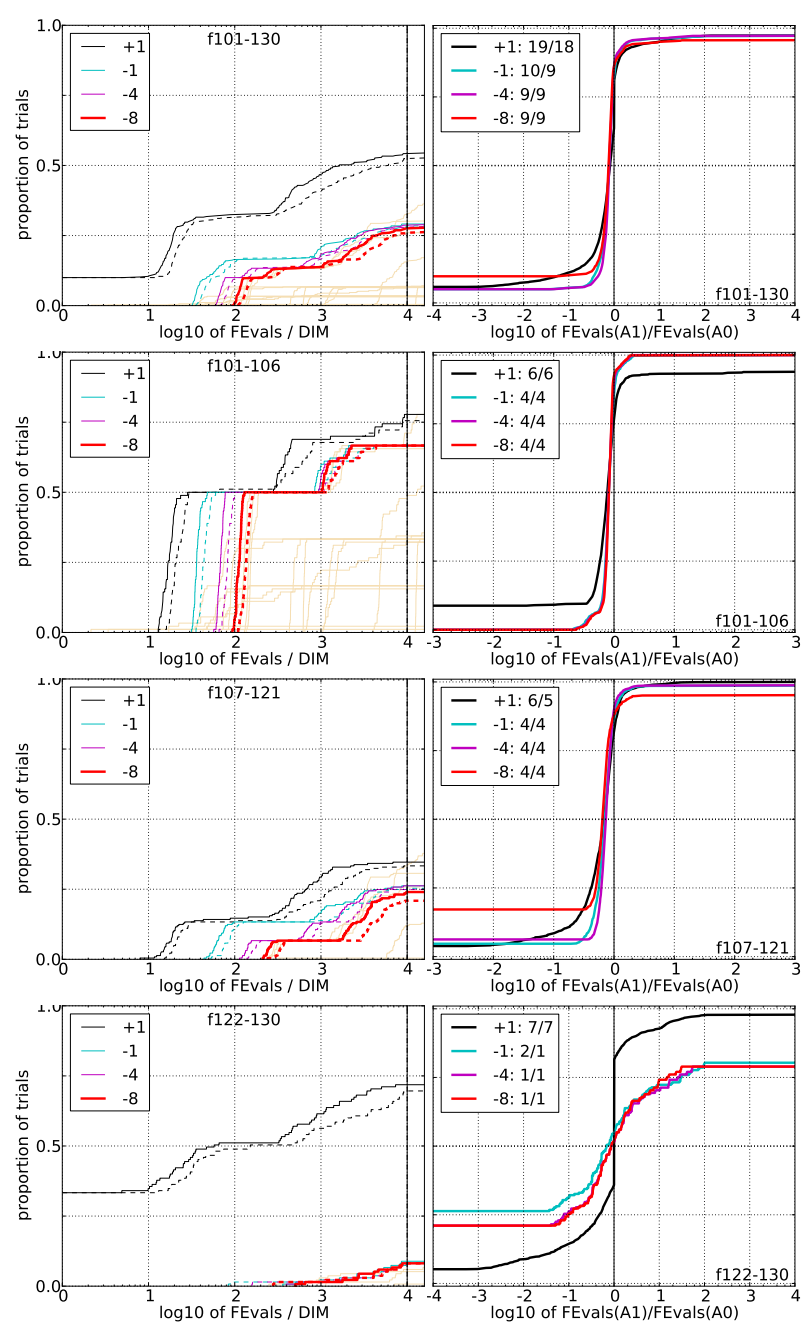

Figure 2: Empirical cumulative distributions (ECDF) of run lengths and speed-up ratios in 5-D (left) and 20-D (right). Left sub-columns: ECDF of the number of necessary function evaluations divided by dimension $D$ (FEvals/D) to reached a target value $f_{\text {opt }}+\Delta f$ with $\Delta f=10^{k}$, where $k \in\{1,-1,-4,-8\}$ is given by the first value in the legend, for $\left(1,4_{\mathrm{m}}\right)$-CMA-ES (solid) and $(1,4)$-CMA-ES (dashed). Light beige lines show the ECDF of FEvals for target value $\Delta f=10^{-8}$ of all algorithms benchmarked during BBOB-2009. Right sub-columns: ECDF of FEval ratios of $\left(1,4_{\mathrm{m}}\right)$-CMA-ES divided by $(1,4)$-CMA-ES, all trial pairs for each function. Pairs where both trials failed are disregarded, pairs where one trial failed are visible in the limits being $>0$ or $<1$. The legends indicate the number of functions that were solved in at least one trial $\left(\left(1,4_{\mathrm{m}}\right)-\mathrm{CMA}-\mathrm{ES}\right.$ first $)$. 
101 Sphere (moderate) 104 Rosenbrock (moderate)

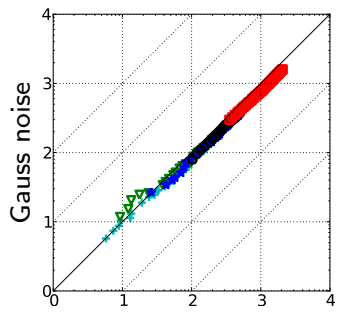

102 Sphere (moderate)

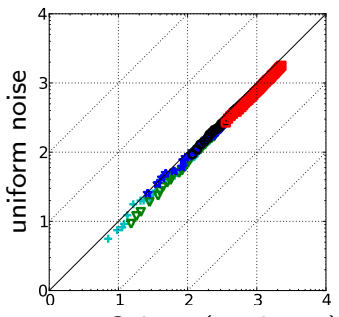

103 Sphere (moderate)

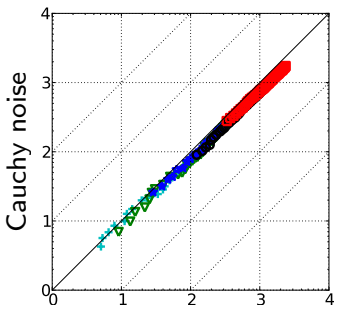

116 Ellipsoid

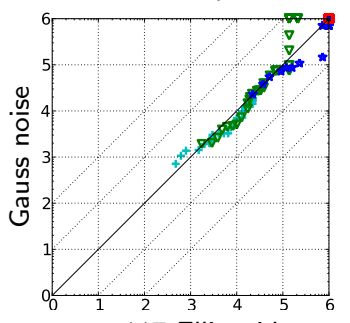

117 Ellipsoid

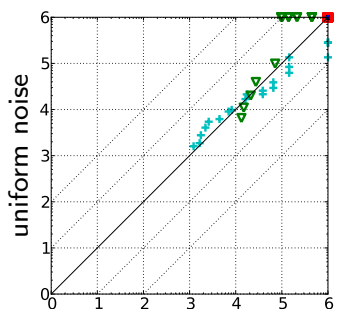

118 Ellipsoid

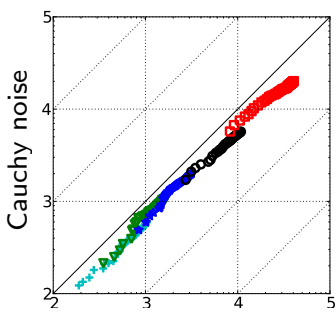

107 Sphere

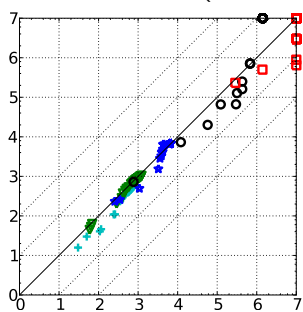

105 Rosenbrock (moderate)

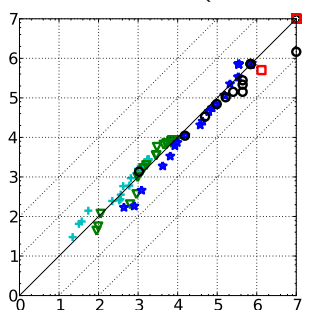

106 Rosenbrock (moderate)
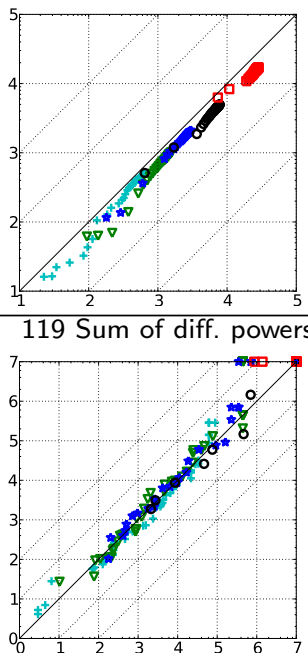

120 Sum of diff. powers

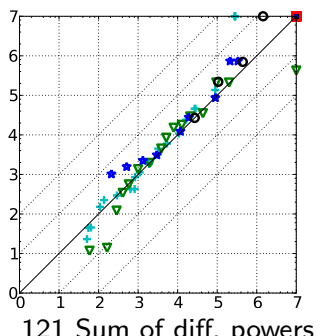

121 Sum of diff. powers

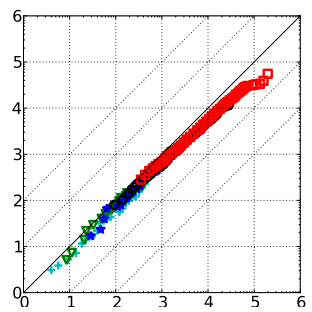

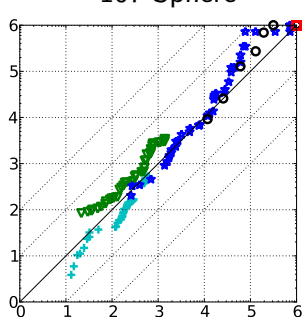

108 Sphere

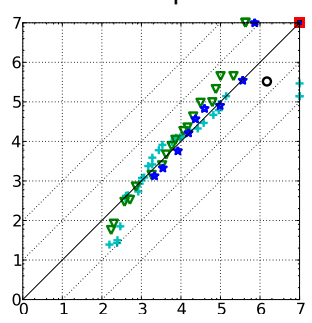

109 Sphere

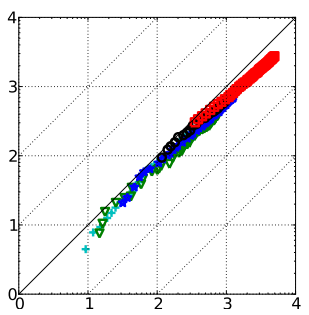

122 Schaffer F7

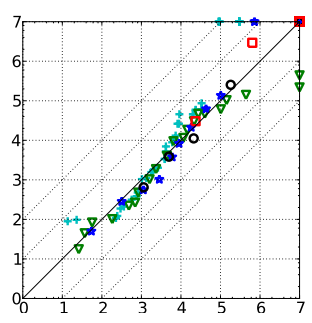

123 Schaffer F7

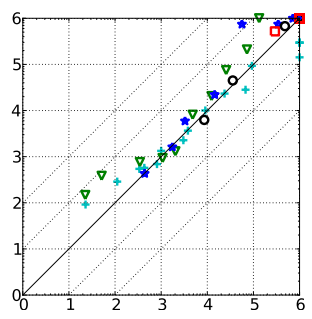

124 Schaffer F7

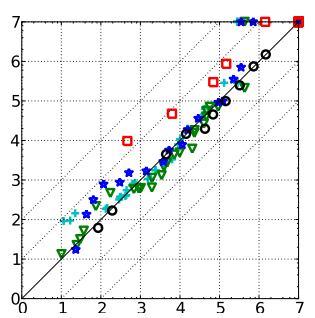

110 Rosenbrock

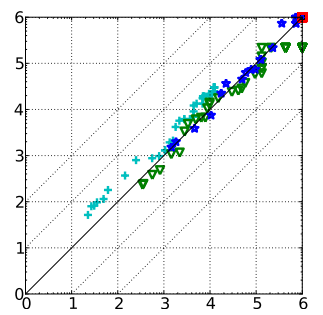

111 Rosenbrock

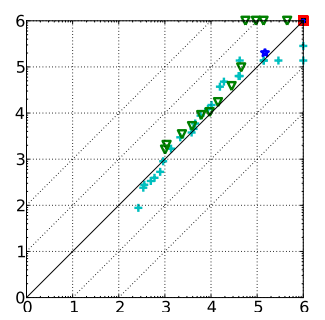

112 Rosenbrock

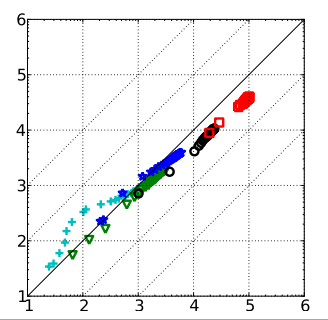

125 Griewank-Rosenbrock

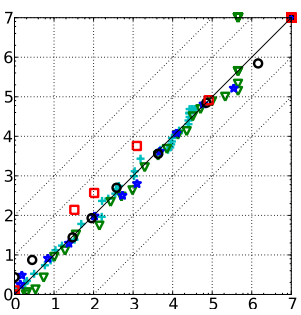

126 Griewank-Rosenbrock

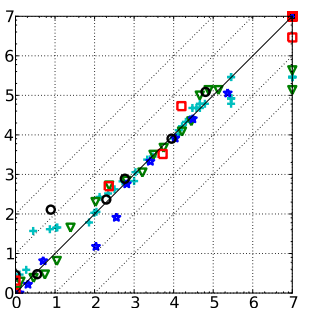

127 Griewank-Rosenbrock

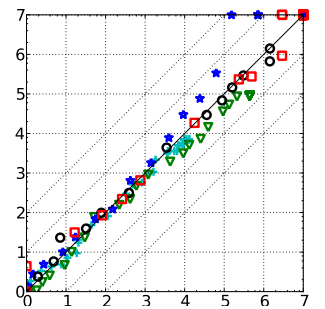

113 Step-ellipsoid

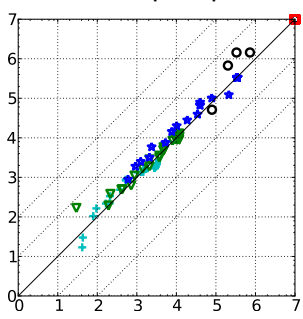

114 Step-ellipsoid

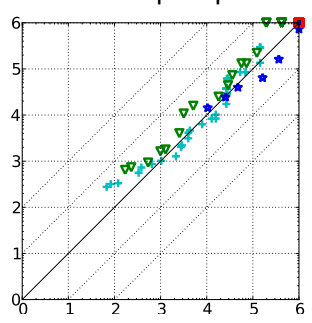

115 Step-ellipsoid

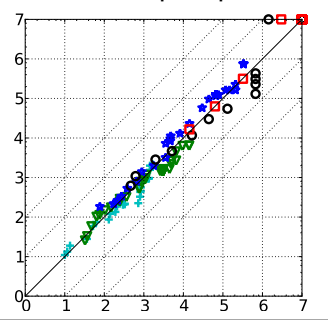

128 Gallagher

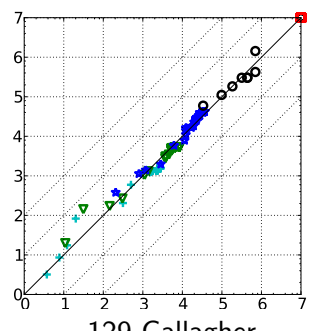

129 Gallagher

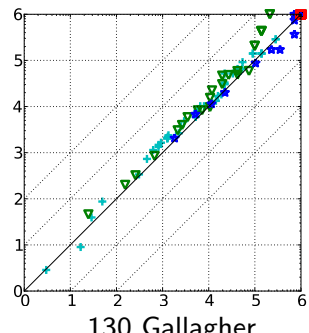

130 Gallagher

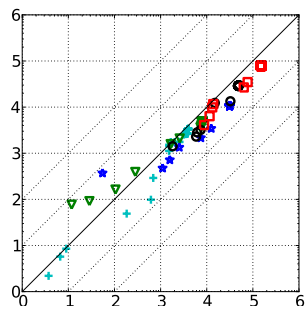

Figure 3: Expected running time (ERT in log10 of number of function evaluations) of (1, $\left.4_{\mathrm{m}}^{\mathrm{s}}\right)$-CMA-ES versus $\left(1,4_{\mathrm{m}}\right)$-CMA-ES for 46 target values $\Delta f \in\left[10^{-8}, 10\right]$ in each dimension for functions $f_{101}-f_{130}$. Markers on the upper or right edge indicate that the target value was never reached by $\left(1,4_{\mathrm{m}}^{\mathrm{s}}\right)-\mathrm{CMA}-\mathrm{ES}$ or $\left(1,4_{\mathrm{m}}\right)-\mathrm{CMA}-\mathrm{ES}$ respectively. Markers represent dimension: 2: $+, 3: \nabla, 5: \star, 10: \circ, 20: \square$. 
$5-\mathrm{D}$
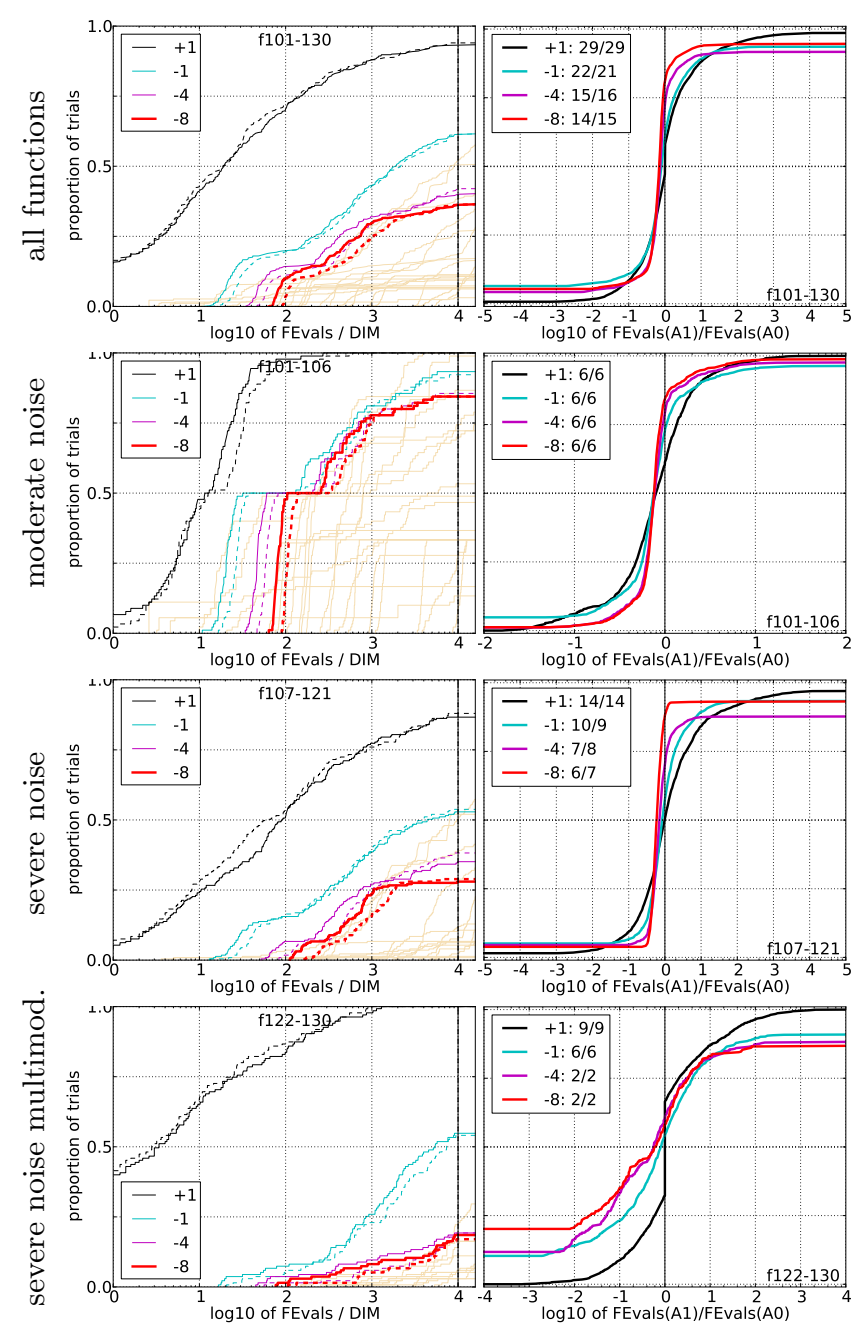

$20-\mathrm{D}$
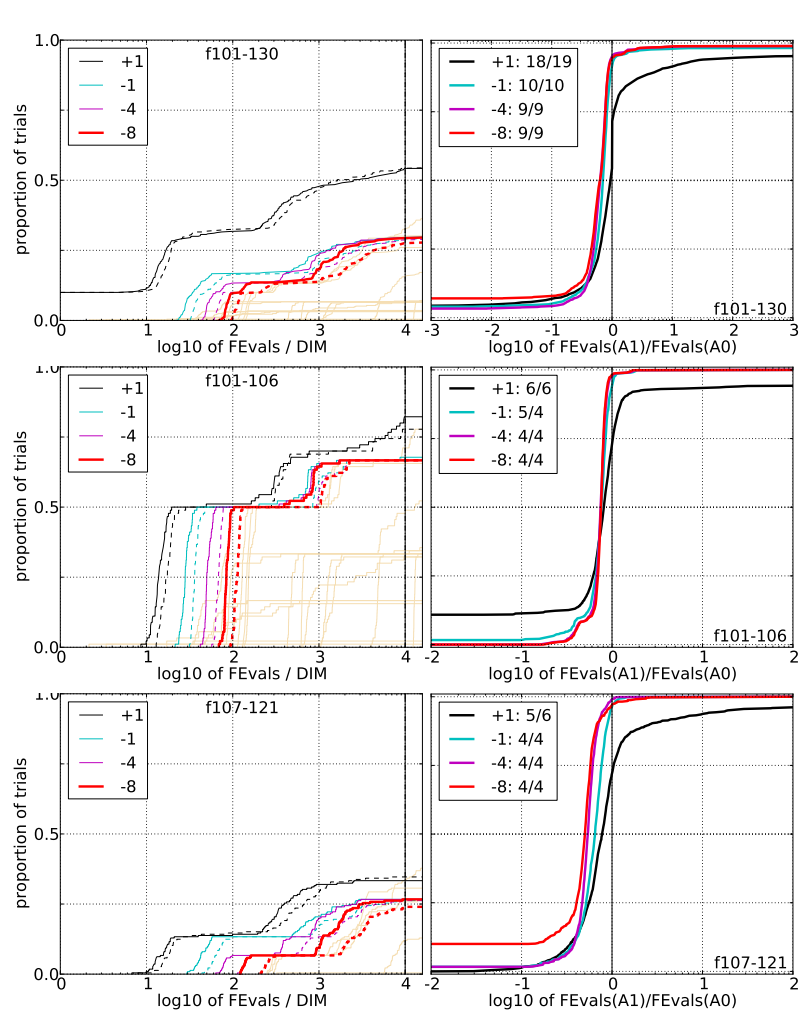

log10 of FEvals(A1)/FEvals(AO)
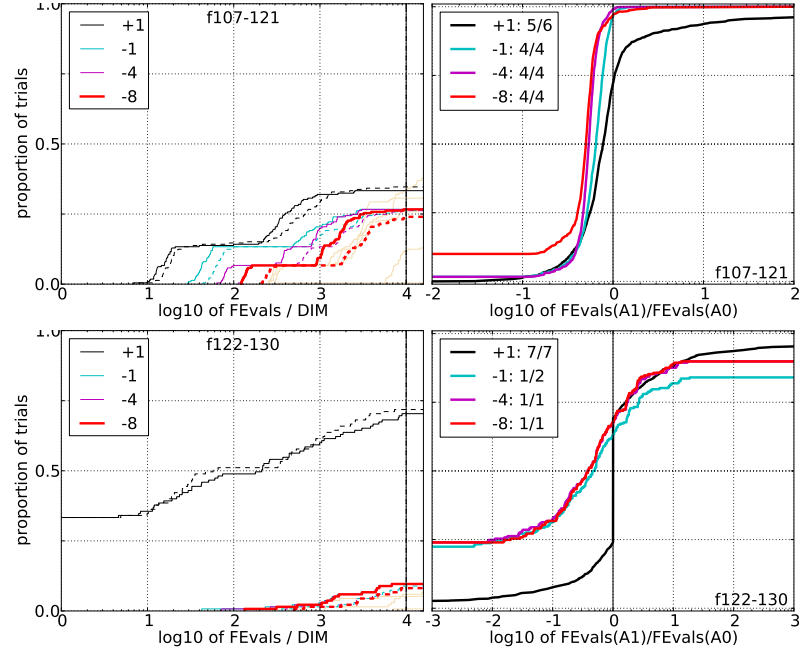

$\log 10$ of FEvals(A1)/FEvals(A0)

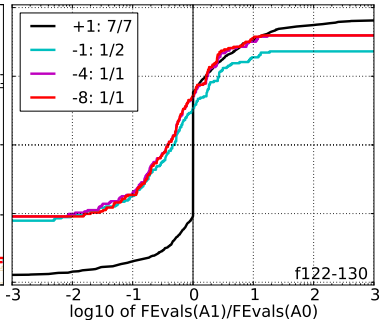

Figure 4: Empirical cumulative distributions (ECDF) of run lengths and speed-up ratios in 5-D (left) and 20-D (right) as in Fig.2 but now for $\left(1,4_{\mathrm{m}}^{\mathrm{s}}\right)$-CMA-ES (solid) and $\left(1,4_{\mathrm{m}}\right)$-CMA-ES (dashed) and ratios of $\left(1,4_{\mathrm{m}}^{\mathrm{s}}\right)$-CMA-ES divided by $\left(1,4_{\mathrm{m}}\right)$-CMA-ES respectively. 
$5-\mathrm{D}$

20-D



Table 2: Expected running time (ERT in number of function evaluations) divided by the best ERT measured during BBOB-2009 as in Table 1 but now comparing $\left(1,4_{\mathrm{m}}\right)$-CMA-ES and $\left(1,4_{\mathrm{m}}^{\mathrm{s}}\right)$-CMA-ES. 